\title{
Clinical practice and randomised controlled trials: There's room for both in individualising patient care
}

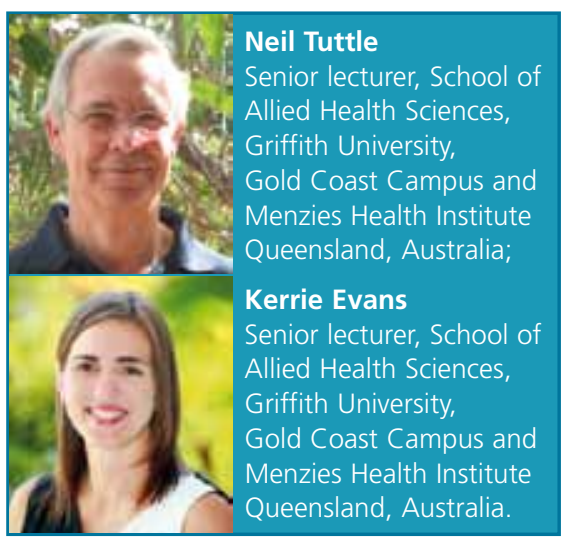

T $\mathrm{t}$ has been suggested that the practice of evidence-based medicine today aims to determine 'the best course of action for this patient, in these circumstances, at this point in their illness or condition' (Greenhalgh et al, 2014). Often, the emphasis in discussions of evidencebased medicine is centred on the interpretation of published evidence. Our intention in this editorial is to discuss how in-depth consideration of the published evidence, judicious use of patterns of treatment, and reassessment can be used to support individualised patient care.

\section{Understanding patient-by- treatment interactions}

Most study designs aim to determine the effectiveness of a given intervention or protocol. Typically, these designs involve recruiting people that meet specific selection criteria before randomly dividing these participants into treatment groups. Now, consider a hypothetical study where patients are divided into four groups receiving different interventions: a control intervention; treatment $\mathrm{A}$; treatment $\mathrm{B}$; and treatments $A+B$ combined (compare with the UK BEAM trial (2004), which had a similar design but smaller effect sizes than will be described below).

The results indicate that the effect size of treatments $A$ and $B$ were approximately 1 , while treatment $\mathrm{A}+\mathrm{B}$ had an effect size of 1.5 (Figures 1 and 2). One interpretation of these findings would be that an individual patient's outcomes would be similar regardless of whether they received treatment A or B. However, another interpretation that is equally consistent with the statistical results is that there are patient-by-treatment interactions.
The concept of patient-by-treatment interactions describes how individuals respond differently to different interventions, and a given individual's response is often distinct from the group response (Senn, 2001). For example, some individuals may have a large improvement with treatment $\mathrm{A}$ but be worse after $\mathrm{B}$, while the reverse would be true for other individuals. The average or group response to the two treatments could therefore be similar, but each individual's response would be more variable in both magnitude and direction.

\section{Predicting treatment response}

Early response to treatment is known to predict future responses to the same treatment. Treatment response may therefore assist in early detection of these non- or negative-responders and enable the treatment to be altered prior to any significant deterioration. This is similar to what clinicians do when applying the methods of clinical decision-making, i.e. using a patient's immediate response following treatment to guide ongoing treatment decisions.

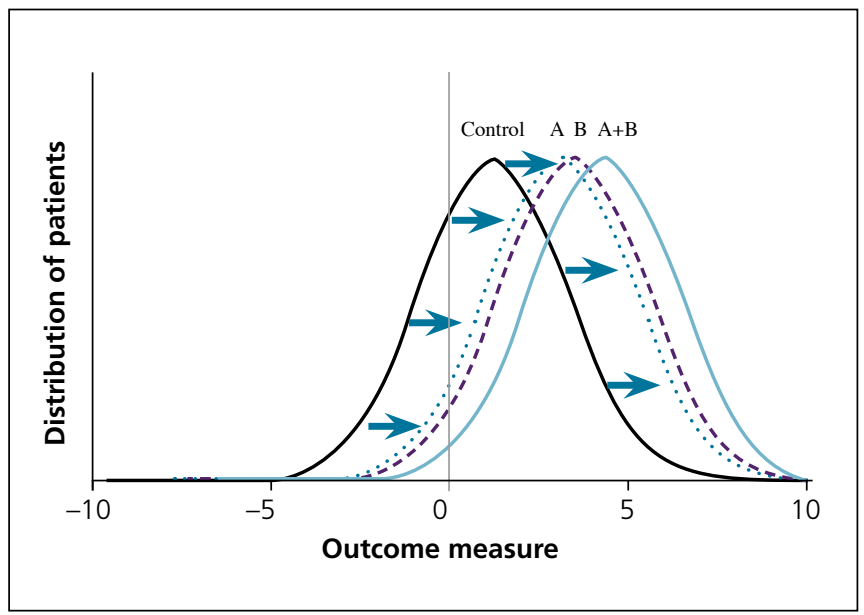

Figure 1. Diagrammatic results from a hypothetical study intended to illustrate the effect of intervention if each individual responded proportionately to the two interventions.

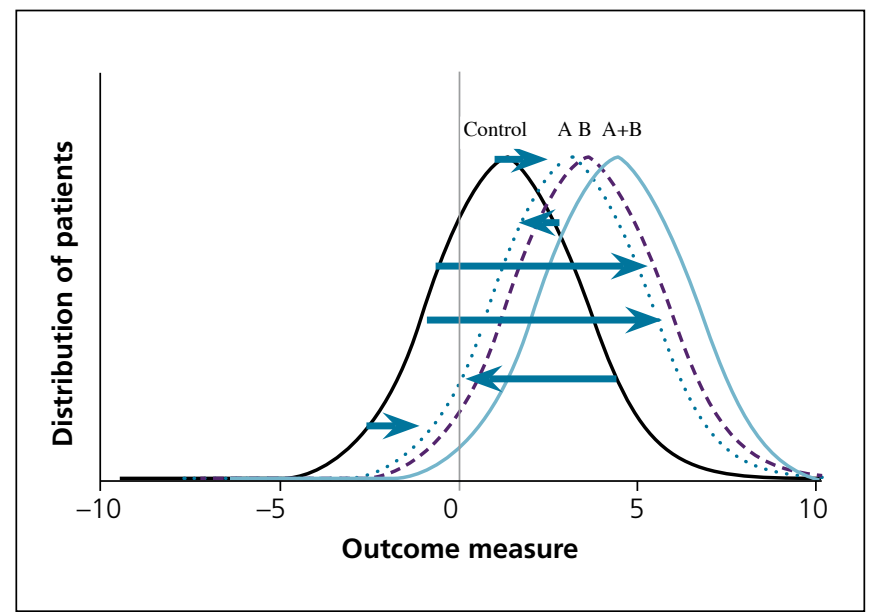

Figure 2. Diagrammatic results from a hypothetical study intended to illustrate an alternative effect from the same findings, where some individuals were better and some worse following intervention. 
There is, however, a classic flaw in the reasoning behind this methodpost hoc ergo propter hoc (after this, therefore because of this)an improvement following one intervention does not necessarily mean the intervention caused the improvement. In addition to this, an immediate change does not indicate that a patient would not respond to a different intervention, and may even respond better to an alternative.

The N-of-1 study design overcomes this flaw by comparing a given patient's response to more than one repetition of each intervention. An application of this method in the clinical setting would be to gauge the response of a given individual to repetitions of at least two interventions in, for example, an $\mathrm{ABAB}$ or $\mathrm{ABBA}$ sequence. The clinician can use the results of this N-of- 1 study to be more confident in their decision to continue with treatment $\mathrm{A}, \mathrm{B}$ or $\mathrm{A}+\mathrm{B}$.

Another innovative study design being used in cancer research is the investigation of 'super-responders', or patients who have an extraordinarily positive response to a given treatment (Dimond, 2014). Researchers then work to determine the characteristics that distinguish these super-responders, such as genetic markers in cancer patients. A version of this process occurs within most clinicians, which, although often referred to as intuition, is largely a recognition of patterns of

\section{'As clinicians working in rehabilitation, we see a diverse group of patients, often with multiple co-morbidities, prescribing treatments that are far from homogenous.'}

signs, symptoms and characteristics that suggest how a patient is likely to respond.

\section{CONCLUSIONS}

As clinicians working in rehabilitation, we see a diverse group of patients, often with multiple co-morbidities, prescribing treatments that are far from homogenous. The evidence for treatment options often does not entirely fit with our patient's clinical presentation and sometimes suggests that multiple treatment options may have similar effects. Even after considering patient preferences and values, it may not be clear which treatment to select. When an intervention is reversible and without conclusive evidence for one intervention over another, there are several strategies available to assist the clinician. These strategies, as discussed earlier, combine clinical judgement and active problem-solving with available evidence to optimise outcomes for individual patients.

UK BEAM Trial Team (2004) United Kingdom back pain exercise and manipulation (UK BEAM) randomised trial: Cost effectiveness of physical treatments for back pain in primary care. BMJ 329: 1381

Dimond PF (2014) Exceptional Responders. The " $N$ of 1 " Paradigm. http://bit. ly/1KnpPzL (accessed 19 May 2015)

Greenhalgh T, Howick J, Maskrey N (2014) Evidence based medicine: A movement in crisis? BMJ 348: g 3725

Senn S (2001) Individual therapy: New dawn or false dawn? Drug Inf J 35(4): 1479-94

\section{Clinical Supervision for Pallative Care}

Clinical supervision has been researched, promoted, and proven as a very effective strategy for constantly developing skills, for maintaining and raising standards, for encouraging personal and professional development, and for building team ethos. This book explores clinical supervision and the qualities, skills, models and ethics needed to ensure success.

Clinical Supervision for Palliative Care has been written as a workbook. You will find pauses for reflection and lots of questions to consider and answer. Good Palliative Care is based on good communication - so you are asked to interact with the text.

Researched with a range of professionals working in the field, and with some of those receiving their care, this book encourages implementation of Clinical Supervision across the widening field of Palliative Care - not only because it will support and sustain practitioners, who are not always good at looking after themselves, but because it will also fulfil the ultimate goal of all of us - the best possible care of dying people, helping them to the death that they want, and easing their suffering and that of those closest to them.

ISBN-13: 978-1-85642-291-8; 234 x 156 mm; paperback; 144 pages; publication 2006; £24.99 